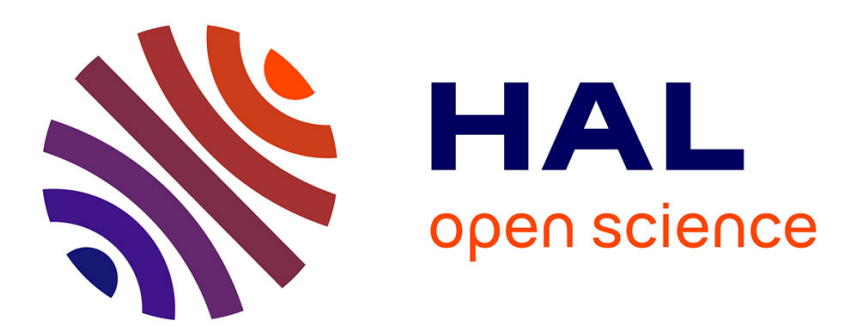

\title{
Improving spatio-temporal resolution of infrared images to detect thermal activity of defect at the surface of inorganic glass
}

G. Corvec, Eric Robin, Jean-Benoit Le Cam, Jean-Christophe Sangleboeuf, Philippe Lucas

\section{To cite this version:}

G. Corvec, Eric Robin, Jean-Benoit Le Cam, Jean-Christophe Sangleboeuf, Philippe Lucas. Improving spatio-temporal resolution of infrared images to detect thermal activity of defect at the surface of inorganic glass. Infrared Physics and Technology, 2016, 77, pp.193-202. 10.1016/j.infrared.2016.05.026 . hal-01372804

\section{HAL Id: hal-01372804 https://hal-univ-rennes1.archives-ouvertes.fr/hal-01372804}

Submitted on 7 May 2020

HAL is a multi-disciplinary open access archive for the deposit and dissemination of scientific research documents, whether they are published or not. The documents may come from teaching and research institutions in France or abroad, or from public or private research centers.
L'archive ouverte pluridisciplinaire HAL, est destinée au dépôt et à la diffusion de documents scientifiques de niveau recherche, publiés ou non, émanant des établissements d'enseignement et de recherche français ou étrangers, des laboratoires publics ou privés. 


\title{
Improving spatio-temporal resolution of infrared images to detect thermal activity of defect at the surface of inorganic glass
}

\author{
Guillaume Corvec $^{a}$, Eric Robin ${ }^{\mathrm{a}, *}$, Jean-Benoît Le Cam ${ }^{\mathrm{a}}$, Jean-Christophe Sangleboeuf ${ }^{\mathrm{a}}$, Pierre Lucas $^{\mathrm{b}}$ \\ ${ }^{a}$ Université de Rennes 1, Institut de Physique, UMR 6251, CNRS/Université de Rennes 1, Campus de Beaulieu, Bât. $10 B$, 35042 Rennes Cedex, France \\ ${ }^{\mathrm{b}}$ Arizona Materials Laboratory, 4715 East Fort Lowell Rd, Tucson, AZ 85712, USA
}

\begin{abstract}
This paper proposes a noise suppression methodology to improve the spatio-temporal resolution of infrared images. The methodology is divided in two steps. The first one consists in removing the noise from the temporal signal at each pixel. Three basic temporal filters are considered for this purpose: average filter, cost function minimization (FIT) and short time Fast Fourier Transform approach (STFFT). But while this step effectively reduces the temporal signal noise at each pixel, the infrared images may still appear noisy. This is due to a random distribution of a residual offset value of pixels signal. Hence in the second step, the residual offset is identified by considering thermal images for which no mechanical loading is applied. In this case, the temperature variation field is homogeneous and the value of temperature variation at each pixel is theoretically equal to zero. The method is first tested on synthetic images built from infrared computer-generated images combined with experimental noise. The results demonstrate that this approach permits to keep the spatial resolution of infrared images equal to 1 pixel. The methodology is then applied to characterize thermal activity of a defect at the surface of inorganic glass submitted to cyclic mechanical loading. The three basic temporal filters are quantitatively compared and contrasted. Results obtained demonstrate that, contrarily to a basic spatio-temporal approach, the denoising method proposed is suitable to characterize low thermal activity combined to strong spatial gradients induced by cyclic heterogeneous deformations.
\end{abstract}

\section{Introduction}

Since the 1980s, infrared measurements have been increasingly used to characterize the mechanical behavior of materials. To date, infrared thermography has proved to be a relevant technique for studying engineering materials such as steel, aluminum and composites. Experiments in this field consist classically in applying a mechanical loading and in measuring the temperature field induced at the material surface by using an infrared camera. Temperature variation field is generally processed from temperature field to determine hydrostatic stress field by using thermoelastic

\footnotetext{
* Corresponding author.

E-mail address: eric.robin@univ-rennes1.fr (E. Robin).
}

stress analysis (TSA) [1], to investigate calorimetric response of materials or to access mechanical dissipation [2]. The results obtained provide information of importance for the understanding of deformation and damage mechanisms such as Luder's bands [3], fatigue $[4,5]$ strain localization [6] or strain-induced crystallization $[7,8]$. Most deformation and damage mechanisms in metals or polymers lead to significant heat production and/or absorption so that the signal to noise ratio in terms of temperature variations is large. Moreover, strain and stress localization generate gradients in the temperature variation field that extend to several infrared pixels. In such conditions, for which infrared data considered are both spatial (infrared image at a given time) and temporal (temperature evolution at each pixel of infrared images), noise pollution does not alter significantly the temperature variation field 
measured. Indeed, the main source of noise is due to infrared detector non-uniformity [9], which is corrected before performing the measurements. Consequently, a spatio-temporal filtering approach permits to obtain satisfactory results. If the signal-tonoise ratio of infrared images in terms of temperature variation becomes low, processing infrared images is more complicated. It is all the more complicated if high temperature variation gradients are induced during the mechanical loading. Recent work in this field has been carried out to characterize temperature variation gradients induced during a cyclic three points bending test applied to soda lime glass [10] and during cyclic compression applied to a chalcogenide glass disk with a hole [11]. Thermal images were processed using average spatio-temporal filtering approach. This method permits to detect temperature variations on the order of one hundredth of a degree and to characterize moderate temperature variations gradients. If higher gradients are generated, for instance in the vicinity of imprints ${ }^{1}$ at the surface of an inorganic glass submitted to mechanical loading, the use of an average filter can smooth or remove the thermal activity in the imprint zone and prevent successful characterization of thermo-mechanical cycles.

The present study aims at proposing a methodology to significantly improve the spatio-temporal resolution of thermal images in challenging conditions. Ideally, the spatial resolution should be one pixel. Such methodology should enable us to quantify low temperature variations and high temperature variation gradients. The paper is structured as follows: Section 2 precisely details the proposed image processing methodology. Section 3 presents the methodology evaluation by means of noised synthetic images. Results are compared with those obtained with the spatiotemporal approach. In Section 4, the methodology is applied to characterize the thermal activity of defect (imprint) at the surface of soda lime glass. Concluding remarks close the paper.

\section{Image processing methodology}

Before detailing the methodology, it is necessary to recall how thermal images are classically obtained in case of mechanical tests. First, the infrared camera is switched on several hours before the beginning of the experiments in order to ensure that its internal temperature is stabilized before performing the calibration and the measurement. The stabilization of the internal temperature of the camera is necessary to avoid any measurement drift during the tests. Second, the Non Uniformity Correction (NUC) of the infrared detector has to be carried out, either by using the manufacturer's protocole or a home-made protocole $[13,14]$. The procedure consists in calibrating the response of each detector of the matrix by considering a black body which surface temperature is homogeneous. Once the mechanical test starts and infrared images are stored, image denoising algorithms consist classically in applying a spatio-temporal filtering: 1 dimension in time, 2 dimensions in space. In this case, the spatial resolution cannot be $a$ priori equal to 1 pixel.

In the present study, images are first filtered temporally. The filtering of temperature variation is carried out independently for each pixel. Once thermal images are filtered temporally, the spatial treatment of images that we propose is based on the thermodynamic principle that if no mechanical loading is applied, no temperature variation can be observed. Therefore, a residual offset can be identified and removed for each thermal pixel by considering thermal images for which no mechanical loading is applied, typically images stored just before starting the mechanical test.

\footnotetext{
1 imprint is obtained after indentation processus that leads to irreversible deformations [12].
}

In such a case, temperature variation field must be homogeneous and the value of temperature variation at each pixel must be equal to zero. In the rest of the paper, the process leading to residual offset removing is called background shifting. Even though final result depends on the efficiency of the temporal filtering, we show in the next section that basic temporal filters already leads to satisfactory results and this make the methodology easy to implement and use.

\section{Evaluation of the proposed methodology}

In the field of image processing, algorithm performances have to be reliably evaluated. Typically in the case of noise suppression algorithms, the procedure consists in comparing an original image and its denoised version. As the original image is unknown, a synthetic image is used to estimate error.

\subsection{Elaboration of noised synthetic images}

At this stage, an important question arises: how to generate representative synthetic noise? In the case of infrared thermography, noise is due to two phenomena. The first one corresponds to photoelectric conversion and interference of signal processing circuits [15]. The second one is induced by experimental conditions, typically convection, radiations and image background. To avoid the development of models taking into account such phenomena, the noise was extracted experimentally from a repeatability test. These measurements were performed in the same conditions as those used for the experimental test described in Section 4 of the present paper. Once the noise was extracted, it was used to build hybrid computer-generated corrupted images.

\subsubsection{Noise extraction with the help of a repeatability test}

The experimental noise was extracted from infrared images during a repeatability test. This repeatability test was then performed at ambient temperature with a black body. The repeatability test consists in measuring the stabilized and homogeneous temperature field at the surface of a black body. Each infrared detector of the camera therefore provides a value of temperature that oscillates around the temperature of the black body. Consequently, extracting the noise means subtracting the average value of the corrupted signal to itself for each pixel. This enabled us to obtain the temporal evolution of the noise generated by the infrared camera considered in this study, which was used to build hybrid computer-generated images. These images were therefore representative of those obtained experimentally.

\subsubsection{Synthetic noised image construction from the repeatability test}

The extracted experimental noise was then added to these synthetic images (see Fig. 1(a)). The goal of this study is to keep 1 pixel spatial resolution after noise suppression. This requires that processed images are not altered by directional effects such as those induced by diagonals and crosses. For example, smoothing a cross leads to a circular shape. This is why the synthetic images in this study simulated local thermal activity of patterns " $\mathrm{X}$ ", "O" and "+", with a width set to 1 pixel. Pixels external to the patterns are set to zero (see pixels in green color in the synthetic image on the left side). The thermal activity of these patterns evolved sinusoidally according to the following equation:

$I(t)=A \cos \left(\frac{2 \pi}{p}\left(t-t_{0}\right)\right)+B$

where $A, B$ and $p$ are amplitude modulation, offset and signal period, respectively. Such signal enables us to simulate thermal response of material under cyclic loading conditions. The signal amplitude modulation $A$ was chosen to be equal to 0.05 , i.e. close to temperature 

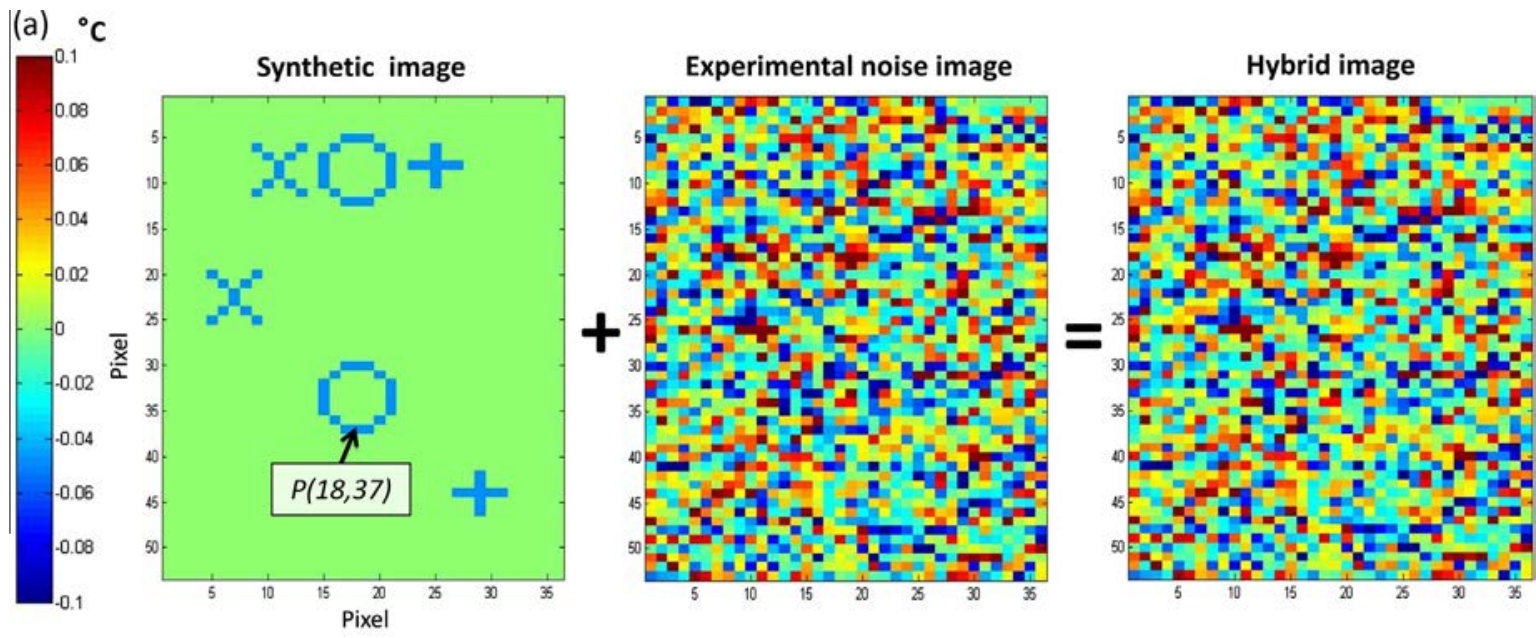

(b) ${ }^{\circ} \mathrm{C}$

Synthetic signal
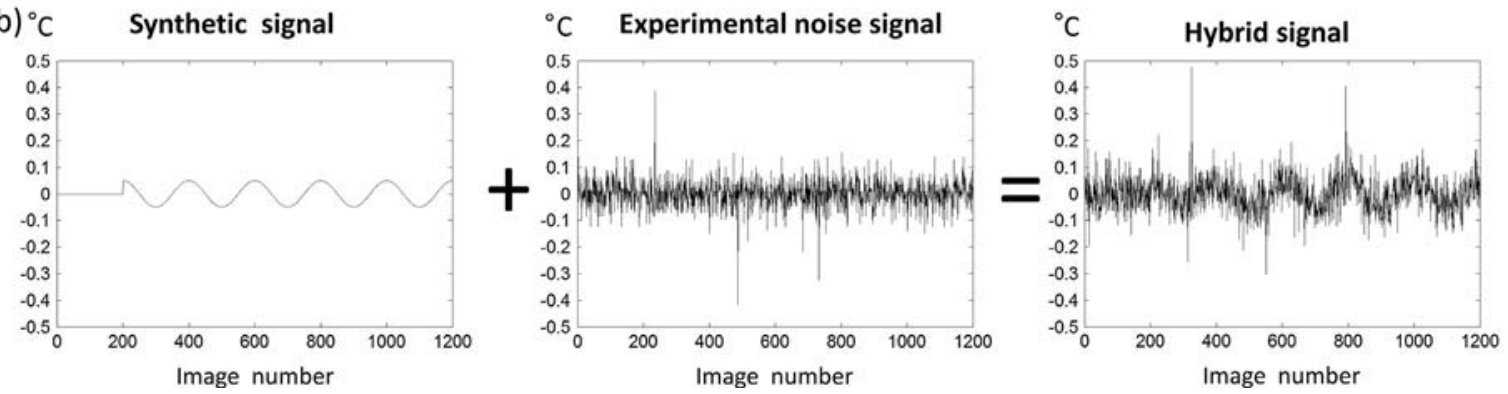

Fig. 1. (a) Generation of hybrid images. (b) Generation of temporal signal at pixel P (see Fig. 1(a)).

variation amplitude found experimentally in a previous study dealing with inorganic glass [10]. $t$ corresponds to the image number and $t_{0}$ corresponds to the value chosen at the beginning of thermal activity. The period $p$ corresponded to 200 frames and is related in practice to the loading frequency. The choice between signal period and frame rate was made with respect to the Shannon sample theorem [16]. The offset was equal to 0 . Fig. 1(b) presents the corresponding temperature variation obtained at pixel $P$ belonging to the pattern. This figure stands from the left to the right side for the imposed temperature (Eq. (1)), the experimental noise and the hybrid signal, which is the combination of the two previous signals.

\subsection{Application to synthetic images}

As mentioned above, the method consists of two steps. The first one corresponds to a temporal filtering, the second one to an offset correction. In practice, any temporal filter can be used. In the present paper, three basic temporal filters were chosen: average filter, cost function minimization (FIT) and short time Fast Fourier Transform approach (STFFT). The objective is to show that even with basic temporal filters, our methodology provides promising results in terms of spatial resolution. Image processing methodology was evaluated with these three filters and results were compared with those obtained with the spatio-temporal approach. First of all, the properties of the three filters used are briefly recalled. Then, denoising methodology is applied. Results obtained are precisely detailed and analyzed.

\subsubsection{First step: temporal filtering}

3.2.1.1. Average filter. The kernel size is defined by the thermal signal frequency. For elastic materials such as inorganic glass, the thermal signal frequency is supposed to be close to the loading frequency. Because the average filters tendency is to reduce the amplitude modulation of the fundamental signal frequency, the user has to carefully choose the kernel size. In our case, a preliminary observation of thermal signal was carried out to determine a suitable kernel size which has to be lower than a quarter of the signal period. This kernel size leads to a filtered signal with sufficient modulation amplitude. Here, the kernel size was set at 41 images.

3.2.1.2. Minimization (FIT). In case of glass materials, which behavior is governed by thermoelasticity, the thermal response varies in phase with the sinusoidal-shaped mechanical loading. A MacLaurin expansion of trigonometric functions leads to polynomial forms of degree $n$. Here, to avoid any oscillation due to noise, $n$ was set to 3 and the kernel size was chosen with respect to the period size, typically one-quarter of the signal period. Here, the kernel size was set at 61 images.

3.2.1.3. Fast Fourier transform. The temporal low pass Fourier filter consists in cutting off high frequencies corresponding to the noise frequencies [17]. In the case of our mechanical tests, low frequency phenomena (such as convection) can also corrupt the original signal, it is therefore more relevant to cut off in terms of short time fast Fourier transform (STFFT) magnitude. First, a short time fast Fourier transform (STFFT) is performed for several pixels at which temperature variation is assumed to be constant. For each temporal signal, i.e. at each pixel, the maximum STFFT magnitude is determined. The cut-off value corresponds to the higher value of maximum STFFT magnitude field. Here, it is equal to $0.019 \mathrm{~dB}$. Second, the STFFT is performed for all the pixels of the temperature variation field. Frequencies corresponding to magnitudes lower than the computed cut-off value are removed. The kernel size must be larger than the signal period. Here, it is set at 201 images. 
3.2.1.4. Results obtained with temporal filters. The three filters described above were applied to hybrid images. Fig. 2 presents the results obtained with these three filters. In this figure, each diagram gives the temperature variation at pixel $\mathrm{P}\left(x_{p}=18, y_{p}=37\right)$ (see Fig. 1(a)) before and after filtering versus the image number. Fig. 2(a), (b) and (c) correspond to FIT, average and STFFT filters, respectively. The original signal is almost retrieved with the three filters and similar results are obtained for the FIT and the average filters. For the FIT and average filters, oscillations are observed either with or without thermal activity. Even though the result obtained in case of the STFFT filter is better (in terms of standard deviation), results are close from one filter to another. Fig. 2(d) gives results obtained with $3 \times 3 \times 41$ spatio-temporal filter. In this diagram, it is clearly observed that the filtered signal is smoothed and its amplitude is lower than obtained in Fig. 2(a)-(c).

\subsubsection{Second step: background shifting}

Background shifting was performed by applying the following processing scheme with Matlab software:

$M_{S}(i, j)=I_{\text {step } 1}\left(i, j, t=t_{0}\right)-I_{\text {step } 1}\left(i=i_{0}, j=j_{0}, t=t_{0}\right)$

where $M_{S}(i, j)$ is the matrix of background shifting. Its components give the difference between the value at the reference pixel in reference image $I_{\text {step } 1}\left(i, j, t=t_{0}\right)$, which coordinates are $i_{0}$ and $j_{0}$, and the value at all the other ones. $t_{0}$ is the time corresponding to the first image of the infrared movie. At $t_{0}$, the value of temperature variation must be equal to zero at any pixel, since no mechanical loading is applied. $I_{\text {step } 1}$ denotes the infrared image at step 1 , meaning after the temporal filtering.

$\forall t>t_{0} I_{\text {step } 2}(i, j, t)=I_{\text {step } 1}(i, j, t)-M_{S}(i, j)-I_{\text {step } 1}\left(i_{0}, j_{0}, t_{0}\right)$

$I_{\text {step2 }}(i, j, t)$ is the result of this image processing methodology.
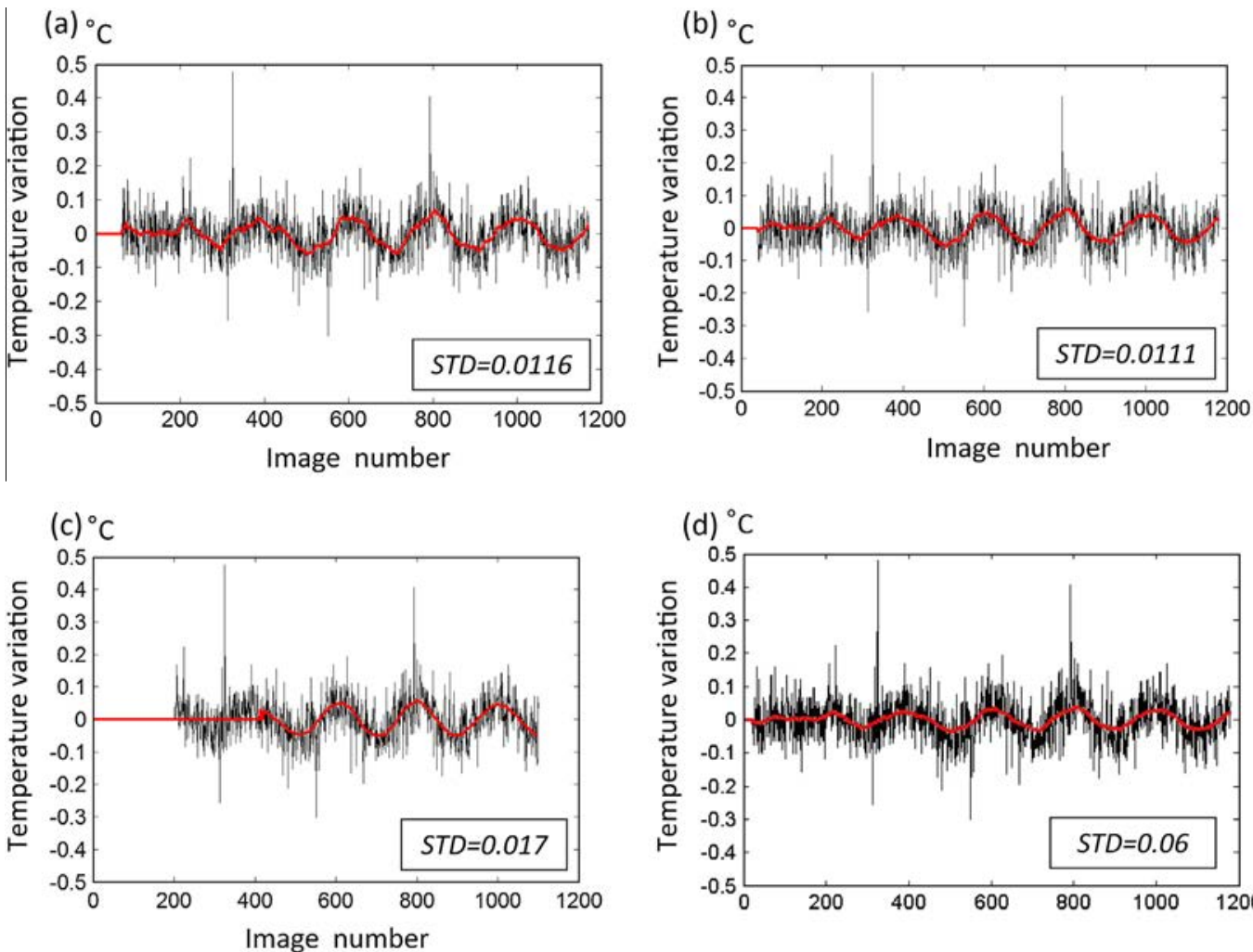

Fig. 3(a), (b) and (c) gives results obtained by applying the background shifting after average, fit and STFFT temporal filtering, respectively. The pattern shape ("X", "O" and "+") is clearly retrieved. This demonstrates that this filtering methodology preserves the full spatial resolution of infrared images even with basic temporal filters. Nevertheless, even with the background shifting, thermal images still appear noisy after the fit filter (Fig. 3(c)) and the average filter (Fig. 3(b)). It is induced by oscillations abovementioned and observed in Fig. 2(a) and (b) (before image number 200). In this paper, temporal filters used are basic ones. It should be noted that if the temporal filter used is more efficient and leads to very low oscillation amplitudes, the background shifting is the equivalent of removing fixed-pattern noise (FPN) [18]. The difference lies in the fact that classically FPN has a spatial structure [19-26], which is not the case here.

Remark. it should be recalled here that such methodology, i.e. filtering only temporally and therefore keeping the spatial resolution to 1 pixel, is made possible because, at a given time before applying the mechanical loading, the value at each pixel is known. Indeed, considering the thermodynamic principle, if no mechanical loading is applied, no temperature variation can be observed. Therefore, a residual offset can be identified and removed for each thermal pixel by considering thermal images for which no mechanical loading is applied, typically images stored just before starting the mechanical test.

\subsubsection{Comparison with spatio-temporal approach}

Hybrid images have been processed by a spatio-temporal approach, more precisely a $3 \times 3 \times 41$ average filter. This filter is the smallest spatial one. It should be noted that this type of filter has previously been used for the detection of thermal activity in

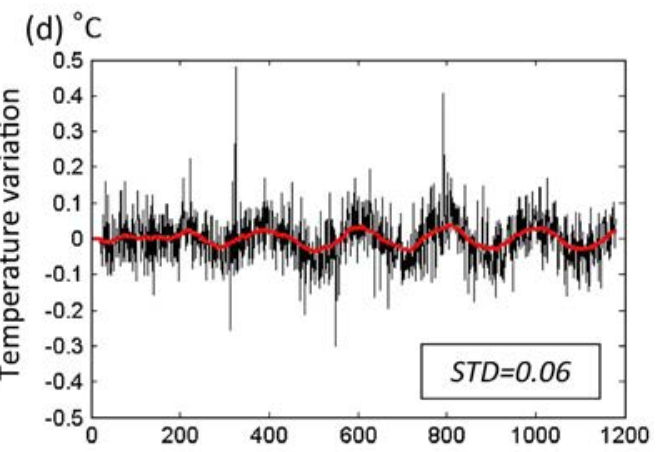

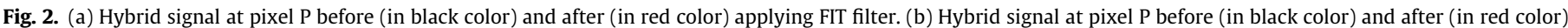

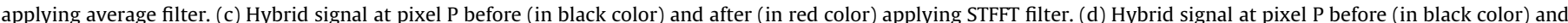

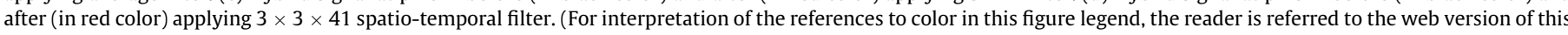
article.) 

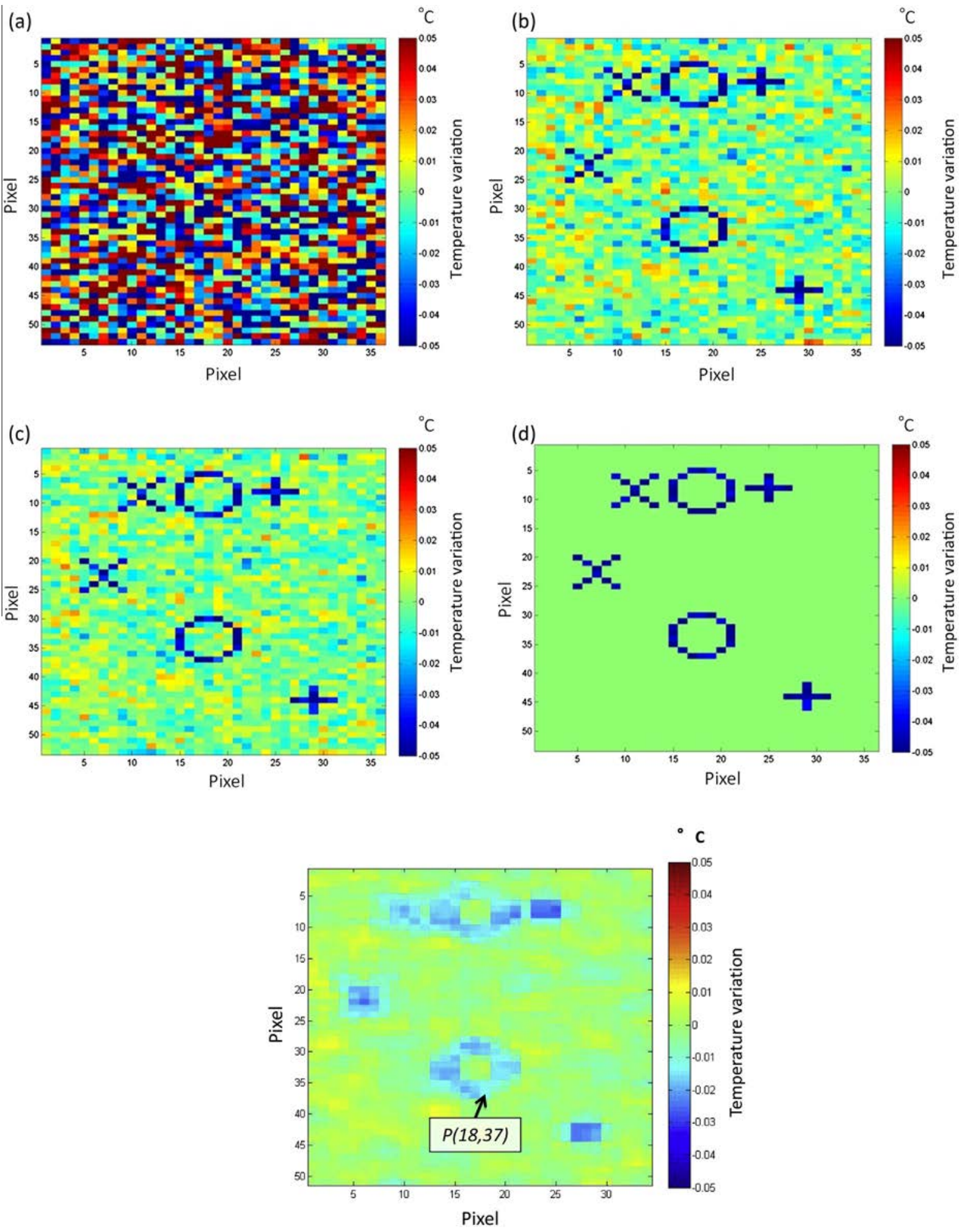

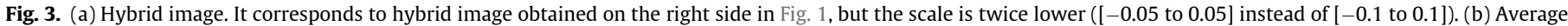
filter. (c) FIT filter. (d) STFFT filter. (e) Spatio-temporal averal filter.

soda lime glasses [10]. The temperature variation field obtained after filtering by this technique is shown in Fig. 3(e). It appears clearly that patterns are smoothed: crosses and diagonal lines are not distinguishable anymore. The spatial resolution is significantly altered by such a filter. Results clearly show that this type of basic filtering is not suitable to process low temperature variations combined with high temperature variation gradients.

\section{Application to the characterization of thermal activity of defect at the surface of soda lime glass}

The full assessment of the proposed noise suppression algorithm cannot only rely on the processing of hybrid infrared images but have to be corroborated with an analysis of experimental infrared images. In this section, we propose to apply our method to the 
detection of the thermal activity of a defect at the surface of soda lime glass submitted to a cyclic loading. Here, thermal activity is characterized thanks to temperature variation maps. To our knowledge, such measurement has never been reported in the literature. The defect corresponds to an imprint issued from indentation. This section is split into two parts. The first part deals with the specimen preparation and the experimental setup. The second part gives the results obtained and their analyze.

\subsection{Experimental set up}

\subsubsection{Material and sample geometry}

Specimen geometry corresponded to a ring of soda lime glass. The specimen was $20 \mathrm{~mm}$ in external diameter, $13 \mathrm{~mm}$ in internal diameter and $2.8 \mathrm{~mm}$ in thickness. The ring was cut with a water jet cutting machine. An imprint was made at its surface with the help of a Matzusawa Vickers indenter. This is illustrated in Fig. 4. The indentation load was set to $50 \mathrm{~N}$. The resulting surface defect was observed with a VHX Keyence microscope. Fig. 5(a) gives a picture of the defect. Fig. 5(b) shows the schematic view of the defect. These figures indicate that radial cracks form in quasiperpendicular directions and chips form in between [12].

\subsubsection{Loading conditions}

The mechanical tests performed was based on the Brasilian test. It is classically used to determine rock tensile strength and to measure rock fracture toughness [27]. In the case of the Brazilian test the specimen geometry is a disk, which was submitted to a compression load, while it is a ring in the present study. It is submitted to cyclic compression loading by means of a 5543 Instron testing machine. An overview of the experimental setup is given in Fig. 6. The signal shape was sinusoidal. The minimum and the maximum values of the force were $-7 \mathrm{~N}$ and $-70 \mathrm{~N}$, respectively. This corresponded to a displacement of $0.07 \mathrm{~mm}$. The sample was submitted to fifteen cycles at a frequency equal to $3.6 \mathrm{~Hz}$.

\subsubsection{Temperature measurement}

Temperature measurements were performed at ambient temperature using the same X6540sc FLIR camera than that used for extracting the noise (section $B$ ). It features a focal plane array of $640 \times 512$ pixels and detectors with a wavelength range of $1.5-$ $5.1 \mu \mathrm{m}$. The integration time was equal to $1000 \mu \mathrm{s}$ and the acquisition frequency was set at $200 \mathrm{fps}$. The resolution is equal to $20 \mathrm{~m} \mathrm{~K}$ at $25^{\circ} \mathrm{C}$, which is approximately equal to room temperature during the test. The thermal emissivity of the material is close to 1 ( $>0.84$ for soda lime glass). The spatial resolution was such that the size of the pixel was equal to $0.015 \mathrm{~mm}$, which is the size of the IR detectors. In order to ensure that the internal temperature of the camera was stabilized before performing the measurements, it was switched on $4 \mathrm{~h}$ before the experiment. Then, the Non
Uniformity Correction (NUC) was performed by using the manufacturer's protocole. Once the temperature field at the specimen surface was stabilized, the temperature measurement was started. Then, the mechanical loading was applied. The test duration was $8 \mathrm{~s}$.

\subsection{Experimental results}

Fig. 7 presents the superposition of temperature variation field, obtained for the maximum of the cyclic force applied to the ring, and elements of the schematic view in Fig. 5(b) in case of $5 \times 5 \times 41$ spatio-temporal filter (Fig. 7(a)), $3 \times 3 \times 41$ spatiotemporal filter (Fig. 7(b)), average filter (Fig. 7(c)), FIT filter (Fig. 7(d)) and STFFT filter (Fig. 7(e)). In all cases, a thermal activity was detected in the imprint zone. As expected for spatio-temporal filters, especially the $5 \times 5 \times 41$ filter, the temperature variation field was smoothed and the imprint morphology was not well detected. The $3 \times 3 \times 41$ filter, which is the smallest spatial one, better highlights the imprint morphology. The maximum level of temperature variation detected increases when the spatial kernel is decreased. Basically for spatio-temporal approaches based on average filter (in time and in space), the lower the spatial kernel size, the higher the weight given to temporal dimension. Consequently, such approach would tend to a purely temporal approach. This is well illustrated by comparing Fig. 7(a) and (b). Fig. 7(c) and (d) presents results obtained by using purely temporal filters issued from the methodology proposed. For average filter and FIT filter, even if the thermal images are strongly noised, the thermal activity zones are not smoothed, consequently, the morphology of the imprint is better detected and the underestimation of the signal level is lower compared to spatio-temporal approach. This shows that even in case of basic temporal filters, satisfactorily results can be obtained with our method. Finally, Fig. 7(e) gives result obtained by using the STFFT filter. As already shown in Section 3 with synthetic images, the spatial resolution is 1 pixel (as the two previous ones), but noise is removed from the thermal field. To assess quantitatively the methodology proposed, Fig. 8 depicts temperature variations along the dotted line in Fig. 7(e) obtained with the three filters and with the spatio-temporal approach. Fig. 8(a), (b) and (c) shows the curve obtained with STFFT filter compared with those obtained with average, fit, $3 \times 3 \times 41$ and $5 \times 5 \times 41$ spatio-temporal filters, respectively. Fig. 8(a) and (b) shows that temperature variations obtained with our methodology fit well with the geometrical singularity at the chip's border. Fig. 8(c) shows that this is not the case for spatiotemporal approach. Even though temperature variation level at the chip's border is similar for $3 \times 3 \times 41$ spatio-temporal and STFFT filters, spatio-temporal filter does not allow to characterize the strong spatial gradient. Obviously, the larger the spatial filter size (here $5 \times 5$ ), the lower the maximum value of temperature

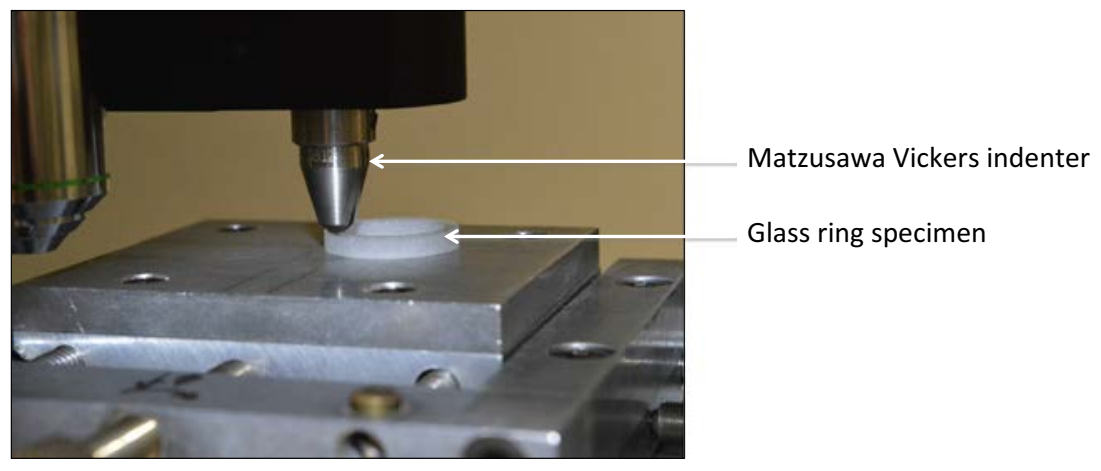

Fig. 4. Indentation with a Matzusawa Vickers indenter. 

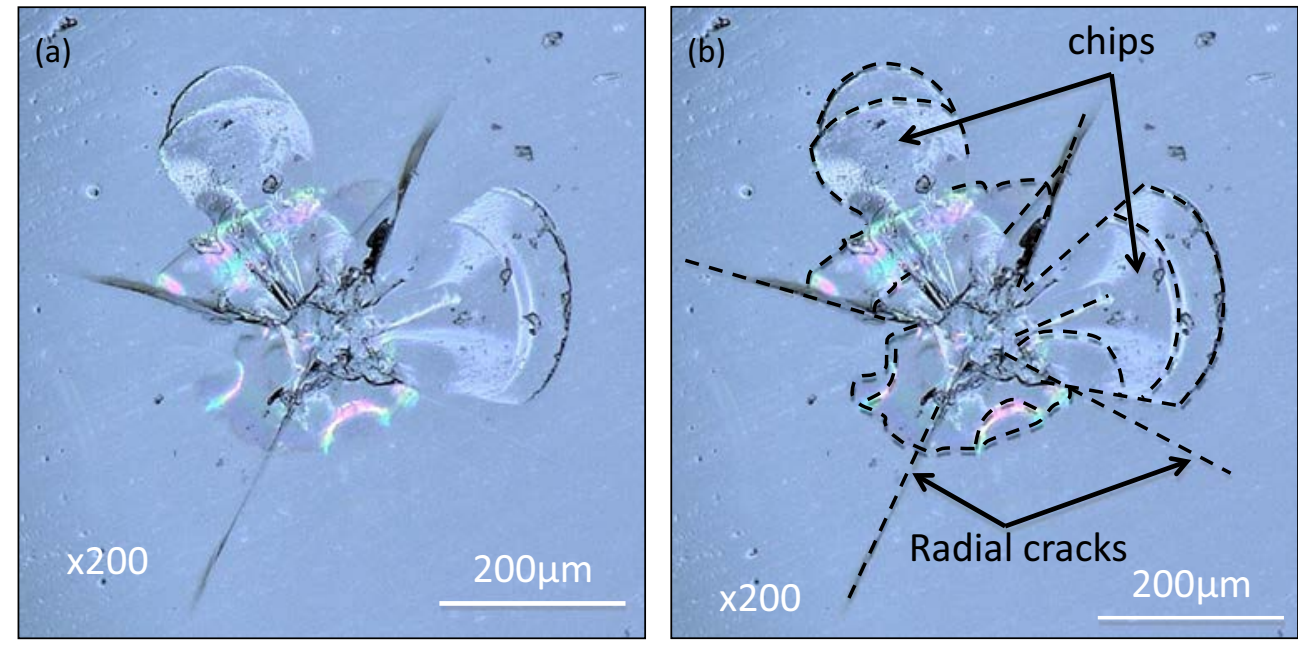

Fig. 5. (a) Imprint performed at $50 \mathrm{~N}$ with a Matzusawa Vickers indenter. (b) Schematic view of indentation zone in Fig. 5(a).

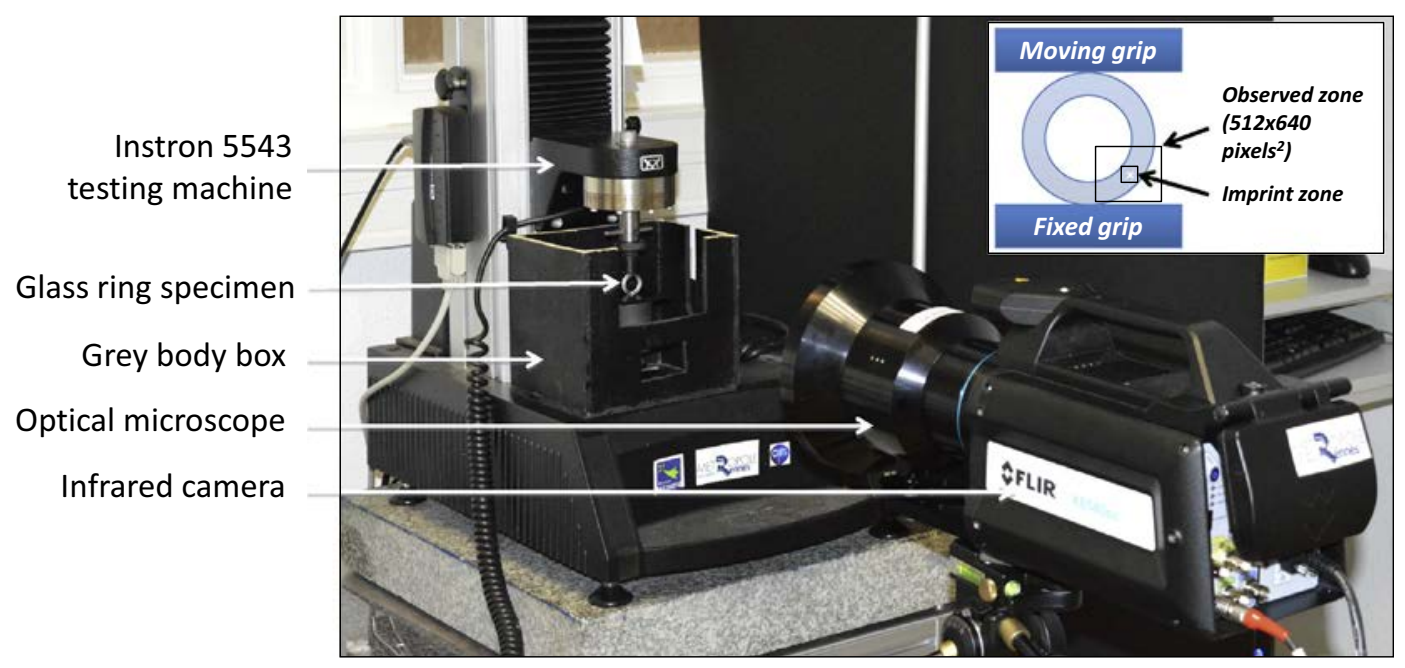

Fig. 6. Overview of the experimental setup.

variation. This clearly shows that spatio-temporal can be used to detect and to quantify temperature variation in case of moderate spatial gradients (see $[10,11]$ ), but not in case of severe spatial gradient such as chip's border in an imprint zone.

These results reveal for the first time the temperature variation map of in imprint zone submitted to mechanical loading and first conclusions on the thermomechanical behavior the imprint zone can also be drawn:

- thermal activity was detected along the chips border, not at the radial crack. The fact that no thermal activity was detected at the tips of the radial cracks does not mean that no stress variation occured at the crack tips. Indeed, temperature variations are due to the variations of the sum of the principal Cauchy stresses, this is the basis of TSA (thermoelastic stress analysis [1]). So, if the mechanical loading induces variation of the sum of the principal Cauchy stresses at the tip of the radial crack, temperature variation will be detected. If the Cauchy stress tensor is mainly composed by a deviatoric part, a stress variation exists but does not induce temperature variation. In the present case, the only conclusion is that there is a variation in the sum of the principal Cauchy stresses at the chip's borders, not at the tip of the radial crack. This is of course related to the mechanical loading applied in the imprint zone. It should be noted that TSA technique is often coupled with photoelasticimetry, which gives the deviatoric components of the Cauchy stress tensor, if optical properties of the material studied allow it (a Brewster constant which ensures a comprehensible fringe pattern). This permits to fully determine the Cauchy stress tensor.

- the blue and red colors are respectively related to negative and positive temperature variations. These temperatures of opposite sign are induced by very different loading conditions, such as typically traction and compression are. Consequently, mechanical field is strongly heterogeneous in the chips zone;

- during the mechanical tests, more specifically during loading and unloading, the temperature variations at the chip borders alternately switch from negative to positive values (equal in absolute value), which is expected in case of elastic deformation of the material;

- at each chip, the thermal activity of some zones is in opposition of phase.

To sum up, the denoising methodology proposed enables us to detect and to quantify temperature variation in zones of high temperature gradient that exhibit low maximum temperature 
(a)

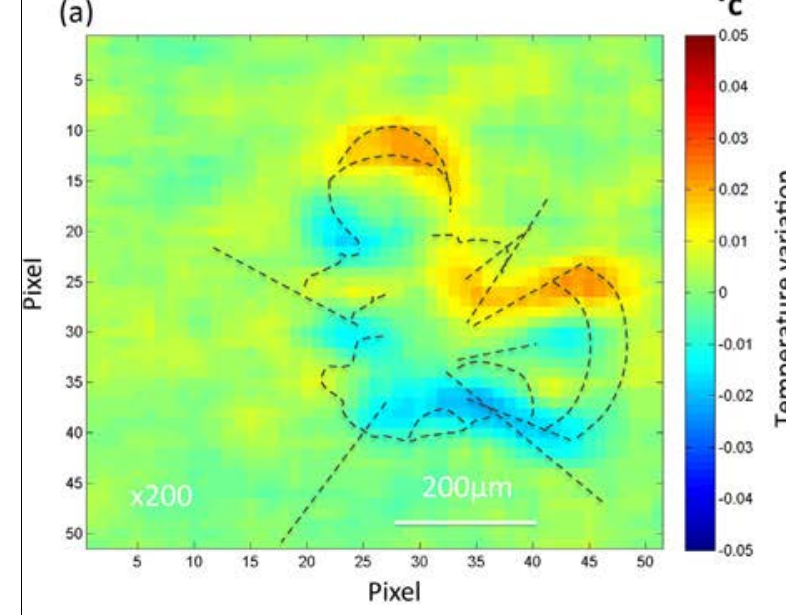

(c)

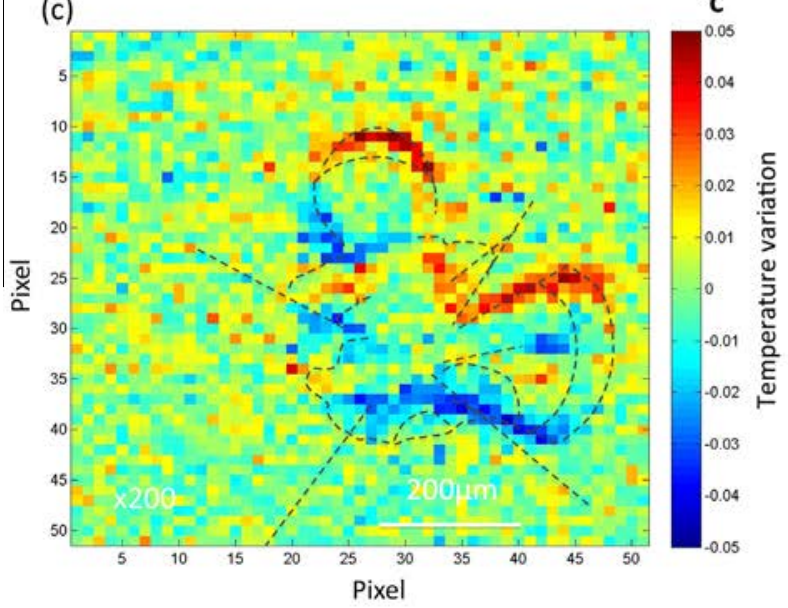

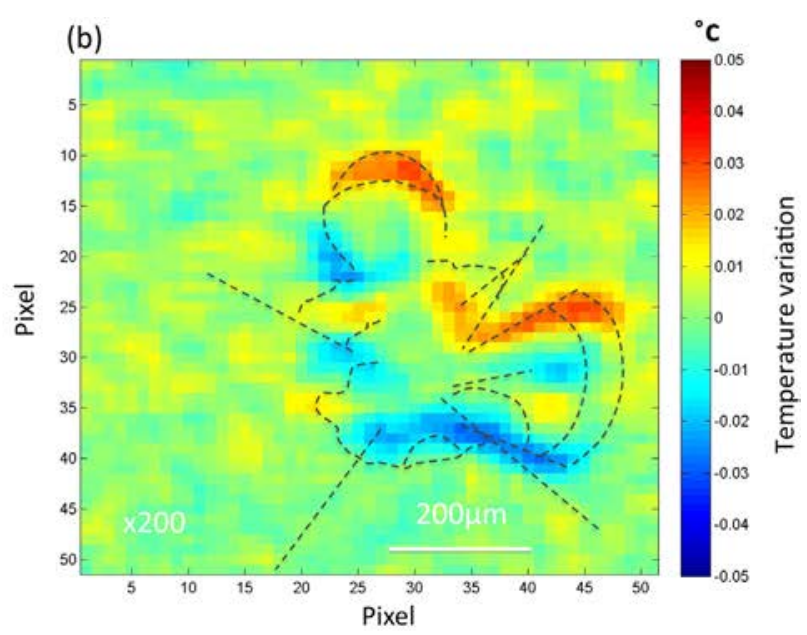

(d)

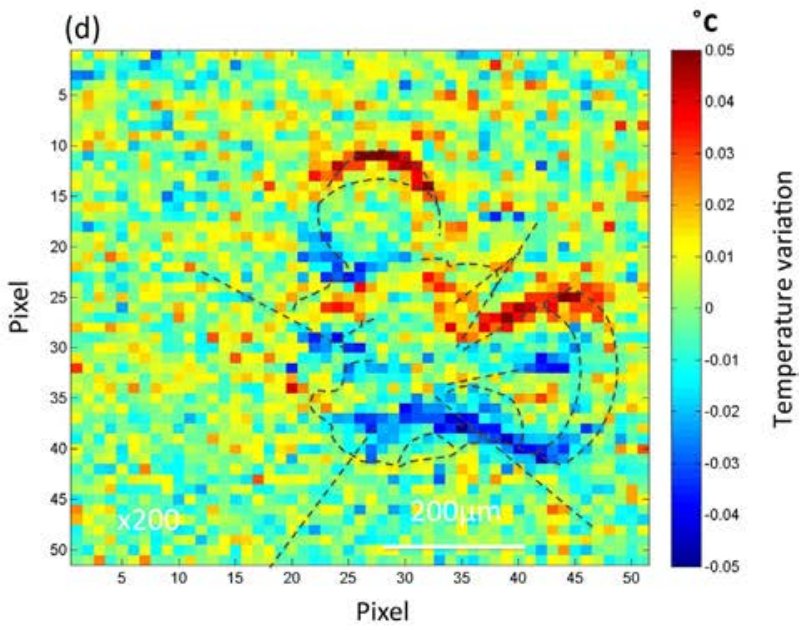

(e)

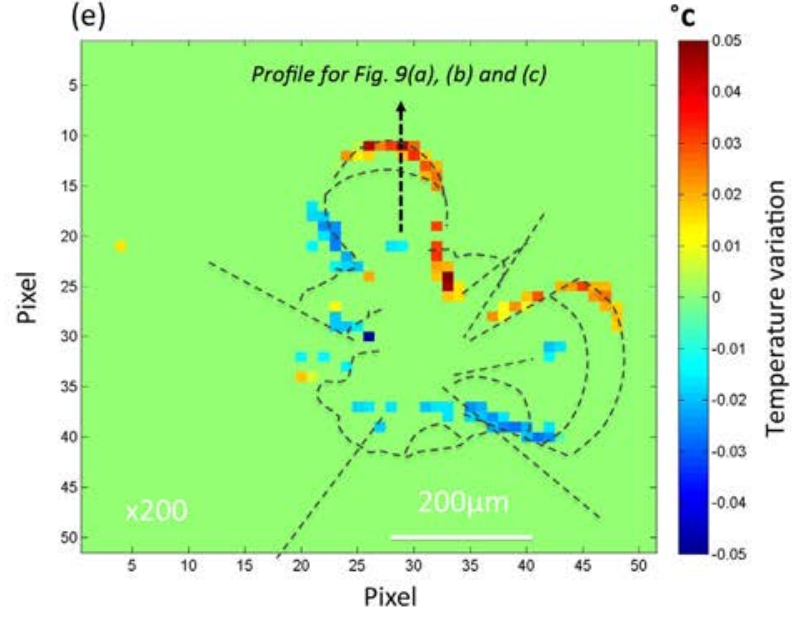

Fig. 7. Thermal activity in the imprint zone (a) $5 \times 5 \times 41$ spatio-temporal filter. (b) $3 \times 3 \times 41$ spatio-temporal filter. (c) average filter. (d) FIT filter. (e) STFFT filter.

variation. This opens a new route for non-destructive testing of glass materials, typical for thermoelastic stress analysis [1] and quantitative calorimetry [6].

\section{Conclusions and perspectives}

In this study, a denoising methodology has been proposed to improve the spatial resolution of infrared images. The methodology is composed of two steps which consists first in removing the noise of the temporal signal at each pixel and second in shifting the residual offset value, which is different from one pixel to another. This methodology has first been tested on hybrid images built from infrared computer-generated images and extracted experimental noise. Results show that the approach is effective at preserving the spatial resolution of infrared image equal to 1 pixel, contrarily to the traditional spatio-temporal approach. By applying this denoising methodology to an imprint zone at the surface of a soda lime glass submitted to mechanical loading, results showed that thermal activity was detected in the chip zones. Negative and positive temperature variations were measured simulta- 
(a) ${ }^{\circ} \mathrm{C}$

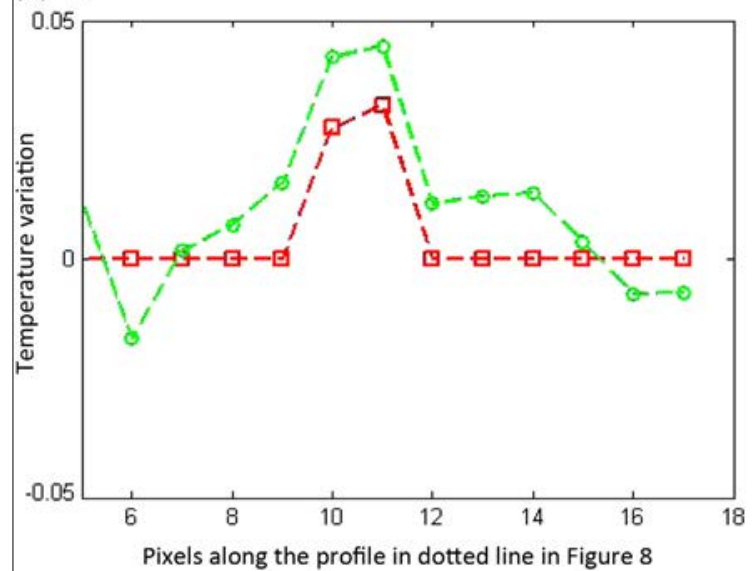

(b) ${ }^{\circ} \mathrm{C}$

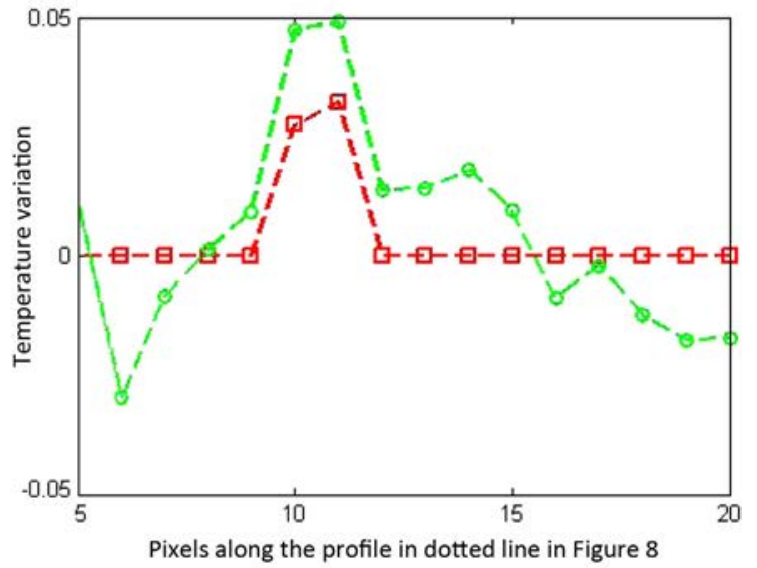

(c) ${ }^{\circ} \mathrm{C}$

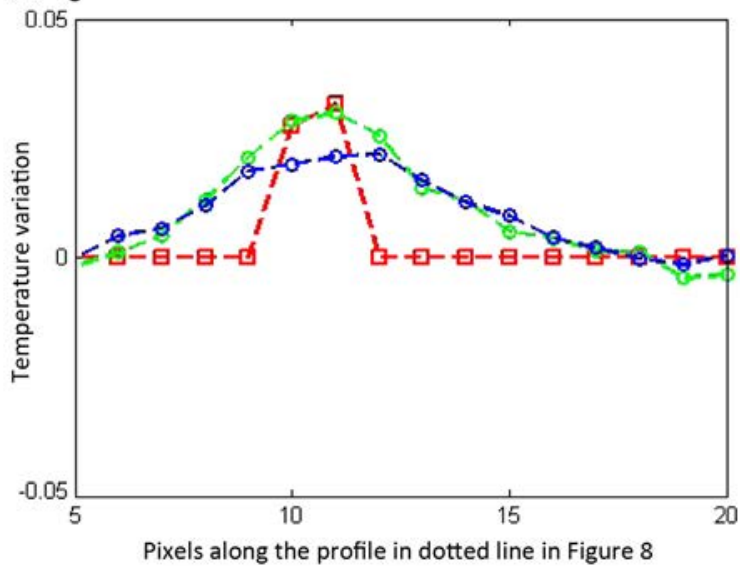

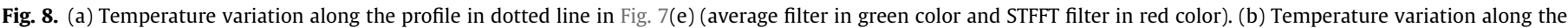

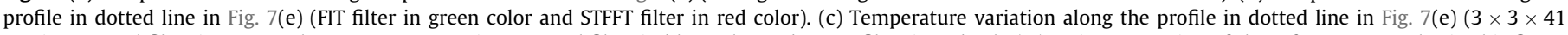

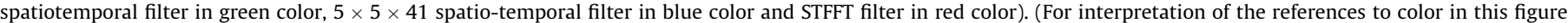
legend, the reader is referred to the web version of this article.)

neously, meaning that at a given time during the mechanical test, chips are submitted to very different loading conditions. The results obtained with this denoising methodology are promising and open new ways of investigation to determine temperature variation fields in case of low signal-to-noise ratio. This is particularly valuable for understanding the thermomechanics of glassy materials, since stresses and heat sources in an imprint zone can henceforth be determined from accurate temperature field measurements.

\section{Funding information}

AIS Scientific Grant from Rennes Métropole (2012).

Mission of Resources and Skills Technology (MRCT) Grant from

French National Center for Scientific Research (2012).

Mission for Interdisciplinary (MI) Grant from French National Center for Scientific Research (2013).

"Politique doctorale" Grant from Rennes 1 University (2013).

\section{Acknowledgment}

The authors thank Dr. Laurent Calvez and Dr. Jean-Pierre Guin for fruitful discussions.

\section{References}

[1] J. Dulieu-Barton, P. Stanley, Development and application of thermoelastic stress analysis, J. Strain Anal. Eng. Des. 33 (1998) 93-104.

[2] A. Chrysochoos, O. Maisonneuve, G. Martin, H. Caumon, J.O, Chezeau, Plastic and dissipated work and stored energy, Nucl. Eng. Des. 114 (1989) 323-333.

[3] A. Chrysochoos, H. Louche, Thermal and dissipative effects accompanying Luders band propagation, Mater. Sci. Eng. A-Struct. 307 (2001) 15-22.

[4] B. Berthel, B. Wattrisse, A. Chrysochoos, A. Galtier, Thermoelastic analysis of fatigue dissipation properties of steel sheets, Strains 43 (2007) 273-279.

[5] M.L. Pastor, X. Balandraud, M. Grédiac, J.L. Robert, Applying infrared thermography to study the heating of 2024-t3 aluminium specimens under fatigue loading, Infrared Phys. Technol. 51 (6) (2008) 505-515.

[6] B. Wattrisse, A. Chrysochoos, J. Muracciole, M. Némoz-Gaillard, Analysis of strain localization during tensile tests by digital image correlation, Exp. Mech. 41 (2001) 29-39.

[7] J.R. Samaca Martinez, J.-B. Le Cam, X. Balandraud, E. Toussaint, J. Caillard, Thermal and calorimetric effects accompanying the deformation of natural rubber. Part 1: Thermal characterization, Polymer 54 (2013) 2717-2726.

[8] J.R. Samaca Martinez, J.-B. Le Cam, X. Balandraud, E. Toussaint, J. Caillard, Thermal and calorimetric effects accompanying the deformation of natural rubber. Part 2: Quantitative calorimetric analysis, Polymer 54 (2013) 27272736.

[9] J. Harris, Y.-M. Chiang, Nonuniformity correction of infrared image sequences using the constant-statistics constraint, IEEE Trans. Image Process 8 (1999) $1148-1151$.

[10] J.-B. Le Cam, E. Robin, X. Balandraud, E. Toussaint, A new experimental route in thermomechanics of inorganic glasses using infrared thermography, J. NonCryst. Solids 366 (2013) 64-69.

[11] E. Robin, J.-B. Le Cam, X. Balandraud, E. Toussaint, L. Brilland, First steps towards the thermomechanical characterization of chalcogenide glass using quantitative infrared thermography, J. Non-Cryst. Solids 391 (2014) 101-105. 
[12] R.F. Cook, G.M. Pharr, Direct observation and analysis of indentation cracking in glasses and ceramics, J. Am. Ceram. Soc. 73 (1990) 787-817.

[13] V. Honorat, Analyse thermomécanique par mesure de champs des élastomères (Ph.D. thesis), Sciences et techniques du Languedoc. Montpellier, Université Montpellier 2 (2006)

[14] M. Poncelet, Multiaxiality, material and stress heterogeneities of self-heating tests and high cycle fatigue tests (Ph.D. thesis), École normale supérieure de Cachan - ENS Cachan, 2007.

[15] D. Scribner, M. Kruer, C. Gridley, K. Sarkady, Measurement, characterization, and modeling of noise in staring infrared focal plane arrays, in: Technical Symposium Southeast, International Society for Optics and Photonics, 1987, pp. $147-160$.

[16] C. Shannon, Communication in the presence of noise, Proc. Inst. Radio Eng. 37 (1949) 10-21.

[17] S. Mohanan, A. Srivastava, Application of the windowed-Fourier-transformbased fringe analysis technique for investigating temperature and concentration fields in fluids, Appl. Opt. 53 (11) (2014) 2331-2344.

[18] F. Perez, J.E. Pezoa, M. Figueroa, S.N. Torres, Empirical frequency domain model for fixed-pattern noise in infrared focal plane arrays, Infrared Phys. Technol. 67 (2014) 413-426.

[19] M. Schulz, L. Caldwell, Nonuniformity correction and correctability of infrared focal plane arrays, Infrared Phys. Technol. 36 (4) (1995) 763-777.

[20] A. El Gamal, B.A. Fowler, H. Min, X. Liu, Modeling and estimation of FPN components in CMOS image sensors, 1998.
[21] J.M. López-Alonso, J. Alda, E. Bernabéu, Principal-component characterization of noise for infrared images, Appl. Opt. 41 (2) (2002) 320-331.

[22] W. Zhao, C. Zhang, Efficient scene-based nonuniformity correction and enhancement, in: IEEE International Conference on Image Processing, 2006 pp. $2873-2876$.

[23] W. Zhao, C. Zhang, Scene-based nonuniformity correction and enhancement: pixel statistics and subpixel motion, J. Opt. Soc. Am. A 25 (7) (2008) 1668 1681.

[24] E. Guadagnoli, C. Giunti, P. Mariani, M. Olivieri, A. Porta, B. Sozzi, S. Zatti, Thermal imager non-uniformity sources modeling, in: Proc. SPIE, 8014, 2011 pp. $80140 \mathrm{~A}-80140 \mathrm{~A}-12$.

[25] O.J. Medina, J.E. Pezoa, S.N. Torres, A frequency domain model for the spatia fixed-pattern noise in infrared focal plane arrays, in: Proc. SPIE, 8155, 2011. pp. $81550 \mathrm{H}-81550 \mathrm{H}-9$.

[26] J.E. Pezoa, O.J. Medina, Progress in pattern recognition, in: Proceedings of Image Analysis, Computer Vision, and Applications: 16th Iberoamerican Congress, CIARP 2011, Pucón, Chile, November 15-18, 2011, Springer, Berlin Heidelberg, 2011, pp. 55-63. Ch. Spectral Model for Fixed-Pattern-Noise in Infrared Focal-Plane Arrays.

[27] J. Henry, Fracture mechanics applied to brazilian tests on disks and rings of rocks, Rendus Hebdomadaires des Séances de l'Académie des Sciences, Serie B 284 (1977) 511-514. 